\title{
Exponentiated uniform distribution: An interesting alternative to truncated models
}

\section{Distribuição uniforme exponenciada: Uma alternativa interessante para modelos truncados}

\author{
Thiago Gentil Ramires ${ }^{1}$; Luiz Ricardo Nakamura ${ }^{2}$; Ana Julia Righetto ${ }^{3}$; \\ Rodrigo Rosseto Pescim ${ }^{4}$; Tiago Santos Telles ${ }^{5}$
}

\begin{abstract}
In this paper some properties of the so-called exponentiated uniform distribution are derived and discussed, such as quantile function, moments, generating function, mean deviations, Bonferroni and Lorenz curves, Shannon and Rényi entropies. The proposed model, defined in the range $[a, b]$ can be used as an alternative to the truncated models. The maximum likelihood estimation of the model parameter is also conducted and a simulation study was performed to verify the consistency of model parameter. An application to a real data set illustrates its potentiality comparing the new distribution with other three well-known truncated distributions. We also present, at the end, an section discussing about computational codes, where the scripts used in R software are available.
\end{abstract}

Keywords: Limited support distributions. Maximum likelihood estimation. Exponentiated distributions.

\section{Resumo}

Neste artigo é apresentado um novo modelo probabilístico denominado de distribuição uniforme exponencial, do qual algumas propriedades da nova distribuição são apresentadas e discutidas, tais como a função quantílica, momentos, função geradora, desvios médios, curvas de Bonferroni e Lorenz, entropia de Shannon e Renyi. O modelo proposto, definido no intervalo $[a, b]$, pode ser utilizado como alternativa aos modelos truncados. A estimativa da máxima verossimilhança do parâmetro do modelo também é realizada e um estudo de simulação foi realizado para verificar a consistência do parâmetro do modelo. Uma aplicação para um conjunto de dados real ilustra a potencialidade do mesmo, comparando a nova distribuição com outras três distribuições truncadas conhecidas na literatura.

Palavras-chave: Distribuições com suporte limitado. Estimação de máxima verossimilhança. Distribuições exponenciadas.

\footnotetext{
${ }^{1}$ Prof. Dr., Dept. Matemática, UTFPR, Apucarana, PR, Brasil; E-mail: thiagogentil@gmail.com

${ }^{2}$ Prof. Dr., Dept. Informática e Estatística, UFSC, Florianópolis, SC, Brasil; E-mail:luiz.nakamura@ufsc.br

${ }^{3}$ Dra., IAPAR, Londrina, PR, Brasil; E-mail: ajrighetto@ gmail.com

${ }^{4}$ Prof. Dr., Dept. Estatística, UEL, Londrina, PR, Brasil; E-mail: rrpescim@uel.br

${ }^{5}$ Dr., IAPAR, Londrina, PR, Brasil; E-mail: telles@iapar.br
} 


\section{Introduction}

A large number of new distributions that extend wellknown distributions and provides great flexibility in modeling data are being investigated in the last years. In this context, (GUPTA et al., 1998) first proposed a generalization of the standard exponential distribution, called the exponentiated exponential (EE) distribution. For a full discussion and some of its mathematical properties, see (GUPTA; KUNDU, 2001). In a similar perspective, (NADARAJAH; KOTZ, 2006b) proposed the exponentiated gamma (EG), exponentiated Fréchet (EF) and exponentiated Gumbel (EGu) distributions, although the way they defined the cumulative density function (cdf) of the last two distributions is slightly different.

In many cases, the properties of these well-known families of distributions can be expressed in terms of the exponentiated family, such as, the Zografos-BalakrishnanG family (ZOGRAFOS; BALAKRISHNAN, 2009), the Weibull-G family (BOURGUIGNON et al., 2014), the Ristic-Balakrishnan family (RISTIC; BALAKRISHNAN, 2012), the Kummer beta generalized family (PESCIM et al., 2012), the exponentiated generalized family (CORDEIRO et al., 2013), among others. Hence, it is extremely important to study exponentiated distributions in order to provide a simpler way to derive some properties of more complex distributions.

One of the simplest distributions in statistics is the uniform distribution, which is defined in the interval $[a, b]$, facilitating the adjustment of data with limited support, e.g., final scores of approved students $[6,10]$ in a given subject, height of military (minimum and maximum will depend on the military academy) etc. The main disadvantage of the uniform model, when compared with other models, is that it is equiprobable throughout all range, which limits its use.

In order to provide some flexibility to this distribution, keeping it in a non pre-defined range, (TORABI; MONTAZERI, 2012) proposed the gamma-uniform (GU) model, which is obtained from the gamma class of distributions when the uniform distribution is used as the baseline, and presented some of its properties. The gammagenerated class of distributions consists in adding two parameters $\alpha$ and $\beta$ to the baseline distribution, providing a better control over its skewness and kurtosis. More recently, (ALJARRAH et al., 2014) presented the Weibulluniform $\{\log$-logistic $\}$ (WU $\{L L\}$ ) distribution with some properties and (TORABI; MONTAZERI, 2014) proposed the logistic-uniform (LU) distribution, derived some pro108 perties of this distribution and presented some applications, thus showing its great flexibility. Lee (LEE; WON, 2006) proposed the exponentiated uniform type I (EUI) distribution with support $(0, \theta)$, considering the uniform distribution over $(0, \theta)$ in the exponentiated class of distributions (GUPTA; KUNDU, 2001), and provided explicit expressions for its moments, moments generating function (MGF), estimation of the parameters, reliability and the right-tail probability. As they considered the uniform distribution with parameters zero and $\theta$, the EUI distribution became very limited.

Similar to (LEE; WON, 2006), we propose in this paper a simple model, belonging to the interval $[a, b]$, using the exponentiated class of distributions (GUPTA; KUNDU, 2001), which has cdf expressed by

$$
F(x)=G(x)^{\alpha}
$$

where $G(x)$ represents the baseline cumulative distribution and $\alpha>0$ denotes the shape parameter. In the proposed model, so-called the exponentiated Uniform (EU) distribution, we assume that $G(x)$ is the uniform $(a, b)$ cdf. Furthermore, deriving equation (1), we can easily obtain the probability density function (pdf) of the exponentiated class of distributions, given by

$$
f(x)=\alpha G(x)^{\alpha-1} g(x),
$$

where $g(x)$ is the baseline pdfF.

The rest of this paper is organized as follows: First we define the EU distribution, provide some special cases and present some general properties such as the quantile function, moments, mgf, mean deviations, Bonferroni and Lorenz curves, entropy and maximum likelihood estimation. After, we perform a simulation study. Lastly, an application to real data is presented followed by conclusions.

\section{Exponentiated uniform distribution}

Let $X$ be a random variable which follows an uniform distribution, with pdf and cdf given by $g(x)=(1) /(b-a)$ and $G(x)=(x-a) /(b-a)$, respectively, where $-\infty<a<x<b<\infty$. The EU density function is defined from (2) by taking $g(x)$ and $G(x)$ to be the pdf and cdf of the uniform distribution, respectively. Its pdf is given by

$$
f(x)=\frac{\alpha}{(b-a)^{\alpha}}(x-a)^{\alpha-1},
$$

where $x \in[a, b]$ and $\alpha>0$ is a shape parameter. 
Based on equation (1), the cdf of the EU distribution is given by

$$
F(x)=\left(\frac{x-a}{b-a}\right)^{\alpha}
$$

A random variable having the pdf, equation (3), is denoted by $X \sim E U(\alpha, a, b)$. For $\alpha=1$, we have the uniform distribution with parameters $(a, b)$. Moreover, if $a=0$ and $b=1$, we obtain the beta distribution with parameters $(\alpha, 1)$, which also corresponds to the Kumaraswamy distribution with parameters $(a, 1)$. If $a=0$, we have the $\operatorname{EUI}(\alpha, b)$ and if $X \sim E U(\alpha, 0,1)$ then $-\log (X) \sim$ $\operatorname{Exponential}(a)$.

In the survival analysis context, using (3) and (4), the survival function and hazard rate function (hrf) of the EU distribution are given by

$$
S(x)=\frac{(b-a)^{\alpha}-(x-a)^{\alpha}}{(b-a)^{\alpha}}
$$

and

$$
h(x)=\frac{\alpha(x-a)^{\alpha-1}}{(b-a)^{\alpha}-(x-a)^{\alpha}},
$$

respectively. Plots of the EU density, cumulative and hazard functions for selected values of $\alpha$ are given in Figure 1. Even though its simplicity, Figure 1(c) shows that the EU distribution has a hrf that includes two types of shape forms, i.e., increasing and bathtub, characteristics that many simple models do not accommodate.

\section{Quantile function and median}

The $p t h$ percentile $x_{p}$ is defined by $F\left(x_{p}\right)=u$. Using (4), the quantile function (qf) can be expressed as

$$
x=Q(u)=F^{-1}(u)=(b-a) u^{\frac{1}{\alpha}}+a .
$$

Quantiles of interest can be obtained from (5) by substituting appropriate values for $u$. In particular, the median of $X$ is obtained when $u=0.5$ by

$$
M=\frac{(b-a)}{2^{\frac{1}{\alpha}}}+a .
$$

We can also use (5) for simulating EU random variables by setting $u$ as an uniform random variable on the unit interval $(0,1)$.

\section{Moments}

We obtain the sth ordinary moment of the EU distribution using equation (2) as follows

$$
\mu_{s}^{\prime}=E\left(X^{s}\right)=\alpha \int_{a}^{b} x^{s} g(x) G(x)^{\alpha-1} d x
$$

Figure 1 - Plots of the EU distribution with $a=0, b=5$ and different values of $\alpha$ : (a) pdf, (b) cdf and (c) hrf.

(a)

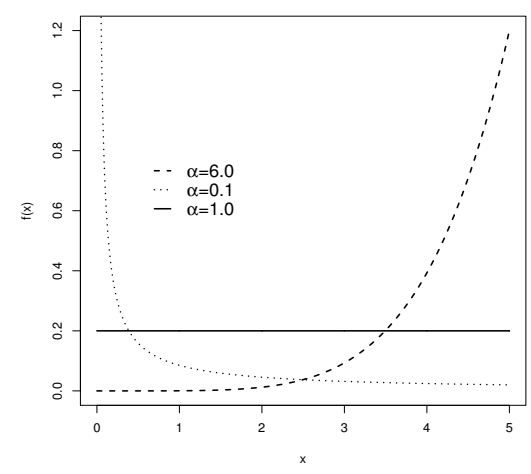

(b)

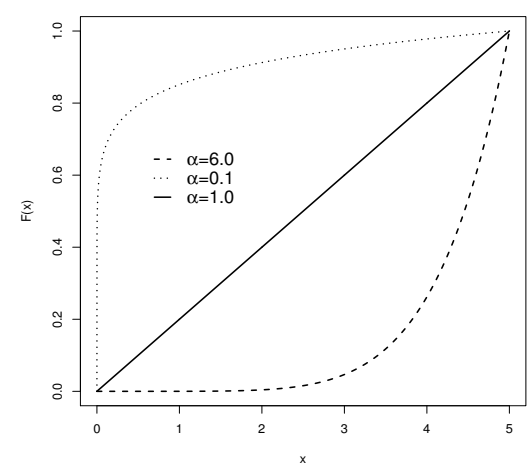

(c)

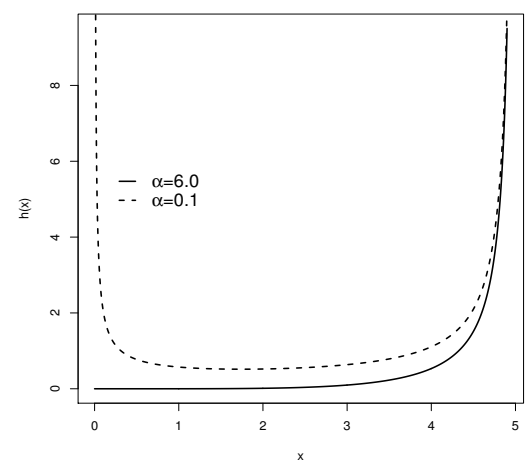

Source: The Authors.

where $G(x)$ and $g(x)$ are the cdf and pdf of the uniform distribution. Setting $u=G(x)$, we obtain

$$
\mu_{s}^{\prime}=\alpha \int_{0}^{1}\left[G^{-1}(u)\right]^{s} u^{\alpha-1} d u,
$$

where $G^{-1}(u)$ denotes the qf of the uniform distribution which can be obtained from (5) when $\alpha=1$. So, the sth moment of the EU distribution is given by

$$
\mu_{s}^{\prime}=\alpha \int_{0}^{1}[(b-a) u+a]^{s} u^{\alpha-1} d u
$$

Using the binomial expansion in equation (6), we obtain

$$
\mu_{s}^{\prime}=\alpha \sum_{k=0}^{s}\left(\begin{array}{l}
s \\
k
\end{array}\right) a^{s-k}(b-a)^{k}(k+\alpha)^{-1} .
$$


The first and second moments, obtained from (7), are given by

$$
E(X)=\frac{\alpha b+a}{\alpha+1}
$$

and

$$
E\left(X^{2}\right)=\frac{2 a^{2}+2 a b \alpha+b^{2} \alpha(\alpha+1)}{(\alpha+1)(\alpha+2)}
$$

respectively. Hence, the variance of the EU distribution becomes

$$
\operatorname{Var}(X)=\frac{\alpha(b-a)^{2}}{(\alpha+1)^{2}(\alpha+2)} .
$$

Plots of mean and variance for some values of $\alpha$ and $b$, considering $a=0$ fixed are displayed in Figure 2 .

Figure 2 - Plots of the (a) mean and (b) variance for the EU distribution with $a=0$ and different values of $\alpha$ and $b$.

(a)

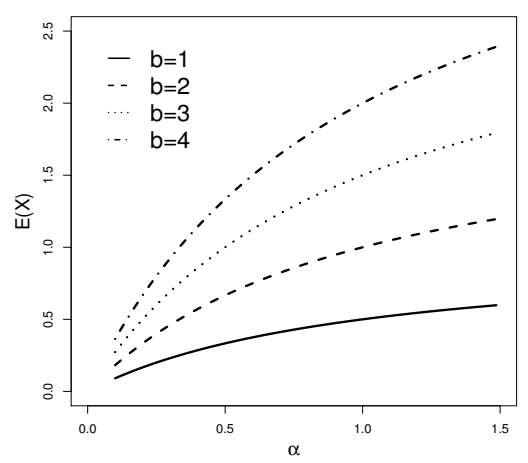

(b)

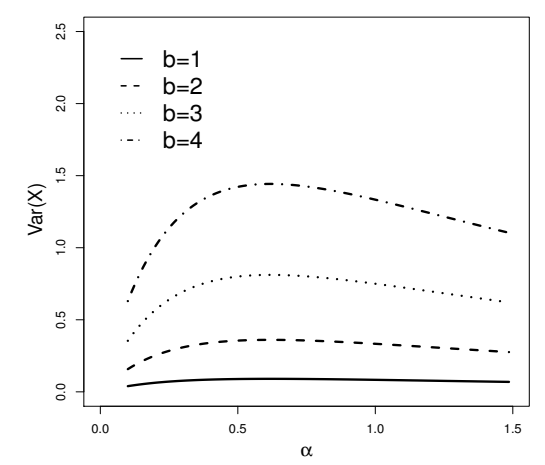

Source: The Authors.

\section{Moment generating function}

Using (2) we provide the formula for the mgf $M(t)=E\left(e^{t X}\right)$ of $X$, where $X \sim \operatorname{EU}(\alpha, a, b)$, as follows

$$
M(t)=\alpha \int_{a}^{b} e^{t x} g(x) G(x)^{\alpha-1} d x
$$

where $G(x)$ and $g(x)$ are the cdf and pdf of the uniform distribution. Considering $u=G(x)$ and computing the integral, the mgf of $X$ reduces to

$$
M(t)=\frac{-\alpha e^{a t} \Gamma[\alpha, t(a-b)]}{[t(a-b)]^{\alpha}},
$$

where $\Gamma(a, z)=\int_{z}^{\infty} t^{a-1} e^{-t} d t$ denotes the incomplete gamma function.

\section{Mean deviations}

The amount of scatter in a population is evidently measured to some extent by the totality of deviations from the mean and the median. If $X$ follows the EU distribution, we can derive the mean deviations about the mean $\mu_{1}^{\prime}=E(X)$ and about the median $M$ from

$\delta_{1}=\int_{a}^{b}\left|x-\mu_{1}^{\prime}\right| f(x) d x=2 \mu[F(\mu)-1]+2 T(\mu)$

and

$\delta_{2}=\int_{a}^{b}|x-M| f(x) d x=2 T(M)-\mu$

respectively, where $T(z)=\int_{z}^{b} x f(x) d x$. The mean $\mu_{1}^{\prime}$ is defined in (7) and the median $M$ is calculated using equation (5) by taking $u=0.5$. The expressions for the mean deviations about the mean and median are given by

$$
\delta_{1}=\frac{2 \alpha^{\alpha+1}(b-a)}{(\alpha+1)^{\alpha+1}}
$$

and

$$
\delta_{2}=\frac{2 a+\alpha a\left(1+0.5^{\frac{1}{\alpha}}\right)+\alpha b\left(1-0.5^{\frac{1}{\alpha}}\right)}{\alpha+1},
$$

respectively (see Appendix A.1).

\section{Bonferroni and Lorenz curves}

The Bonferroni and Lorenz curves have applications not only in economics to study income and poverty, but also in other fields like reliability, insurance, medicine and demography. They are defined by

$$
B(p)=\frac{1}{p E(X)} \int_{a}^{q} x f(x) d x
$$

and

$$
L(p)=\frac{1}{E(x)} \int_{a}^{q} x f(x) d x
$$


respectively, where $q=Q(p)$ is given by (5) for a given probability $p$. Therefore, the curves for $X$ are given by (see Appendix A.2)

$$
B(p)=\frac{\alpha p^{\frac{1}{\alpha}}+a \alpha+a}{\alpha b+a}
$$

and

$$
L(p)=\frac{p\left(\alpha p^{\frac{1}{\alpha}}+a \alpha+a\right)}{\alpha b+a}
$$

\section{Entropies}

Two popular entropy measures are the Rényi (RÉNYI, 1961) and Shannon (SHANNON, 1951) entropies. The Rényi entropy of a random variable with pdf $f(\cdot)$ is defined as

$$
I_{R}(\gamma)=\frac{1}{1-\gamma} \log \int_{-\infty}^{\infty} f^{\gamma}(x) d x
$$

for $\gamma>0$ and $\gamma \neq 1$. The Shannon entropy of a random variable $X$ is defined by $E[-\log f(X)]$. It is the particular case of the Rényi entropy when $\gamma \uparrow 1$.

Using (3) we obtain for the EU distribution

$$
\begin{aligned}
\int_{-\infty}^{\infty} f^{\gamma}(x) d x & =\int_{a}^{b} \frac{\alpha^{\gamma}}{(b-a)^{\alpha \gamma}}(x-a)^{\alpha \gamma-\gamma} d x \\
& =\left[\frac{\alpha^{\gamma}(b-a)^{1-\gamma}}{\gamma(\alpha-1)+1}\right] .
\end{aligned}
$$

Therefore, the Rényi entropy of $X$ is expressed by

$$
I_{R}(\gamma)=\frac{\gamma \log \alpha-\log (\gamma(\alpha-1)+1)}{1-\gamma}+\log (b-a)
$$

The Shannon entropy can be obtained by limiting $\gamma \uparrow 1$ in (13). Using the pdf expressed in (3) we have

$$
E[-\log f(X)]=\log (b-a)-\log (\alpha)+\frac{\alpha-1}{\alpha} .
$$

\section{Inference}

Let $X_{i}$ be a random variable following (3) with vector $\theta=(\alpha, a, b)^{\top}$ of parameters. Note that $a$ and $b$ parameters are fixed according to the data set (minimum and maximum, usually), so only $\alpha$ will be estimated, i.e., $\theta=(\alpha)^{\top}$. The $\log$-likelihood $l(\theta)$ for the model parameter, considering a sample size of length $n$, is given by

$l(\theta)=n[\log (\alpha)-\alpha \log (b-a)]+(\alpha-1) \sum_{i=1}^{n} \log \left(x_{i}-a\right)$.
The score function for parameter $\alpha$ can be obtained from the last eq. and is given by

$$
U_{\alpha}(\theta)=\frac{n}{\alpha}-n \log (b-a)+\sum_{i=1}^{n} \log \left(x_{i}-a\right) .
$$

The maximum likelihood estimate (MLE) $\hat{\theta}$ of $\theta$ is obtained by solving the nonlinear likelihood equation $U_{\alpha}(\theta)=0$. The MLE for $\alpha$ obtained from (14) is expressed by

$$
\hat{\alpha}_{\mathrm{MLE}}=\frac{1}{\log (b-a)-\sum_{i=1}^{n} \log \left(x_{i}-a\right)} .
$$

Considering $a$ and $b$ fixed, for interval estimations and hypothesis tests of the model parameter $\alpha$, we require the unit observed information $J(\alpha)=\left\{L_{\alpha \alpha}\right\}$. Under conditions that are fulfilled for parameters in the interior of the parameter space but not on the boundary, the asymptotic distribution of $\sqrt{n}(\widehat{\alpha}-\alpha)$ is $N\left(0, I(\alpha)^{-1}\right)$, where $I(\alpha)$ is the unit expected information. Based on the the normal $N\left(0, J(\widehat{\alpha})^{-1}\right)$ distribution, where $J(\widehat{\alpha})$ is the observed information matrix evaluated at $\alpha$, we can construct approximate confidence intervals and confidence regions for the parameter $\alpha$, whose element is given

$$
J_{11}=\frac{\partial^{2} \log L}{\partial \alpha^{2}}=\frac{-n}{\alpha^{2}} \text {. }
$$

\section{Simulation}

To perform the simulation study with the EU distribution, we set $\mathrm{U}$ from (5) as an uniform random variable on the unit interval $(0,1)$. We simulate data from an EU, $\alpha=0.5,1,4, a=0, b=1$, distribution and use three different sample sizes $(n=20,50,150$, and 300). For each sample size, we compute the MLEs of $\alpha$. This process was repeated 1,000 times and the average estimates (AE), biases and the mean squared errors (MSEs) are computed. The results are reported in Table 1. The required numerical evaluations are implemented in $\mathrm{R}$ software through the function optim.

Comparing the performance of the estimators in Table 1, we can verify that the MSE values decrease for all scenarios as the sample size increase, indicating a consistence in the MSE.

\section{Application}

In this section we introduce a real data set in order to illustrate the potentiality of the new distribution, comparing its fit with some truncated models. 
Table 1 - AE, bias and MSEs based on 1,000 simulations of the EU distribution for $\alpha=0.5,1$ and 4 , by fixing $a=0$ and $b=1$, for sample sizes $n=20,50,150$ and 300 .

\begin{tabular}{ccccc}
\hline$\alpha$ & $n$ & AE & Bias & MSE \\
\hline 0.5 & 20 & 0.53 & 0.03 & 0.02 \\
0.5 & 50 & 0.51 & 0.01 & 0.01 \\
0.5 & 150 & 0.51 & 0.01 & 0.00 \\
0.5 & 300 & 0.50 & 0.00 & 0.00 \\
\hline 1 & 20 & 1.05 & 0.05 & 0.06 \\
1 & 50 & 1.01 & 0.01 & 0.02 \\
1 & 15 & 1.00 & 0.00 & 0.01 \\
1 & 300 & 1.00 & 0.00 & 0.00 \\
\hline 4 & 20 & 4.18 & 0.18 & 0.95 \\
4 & 50 & 4.10 & 0.10 & 0.36 \\
4 & 15 & 4.02 & 0.02 & 0.12 \\
4 & 30 & 4.02 & 0.02 & 0.06 \\
\hline
\end{tabular}

Source: The Authors.

The original data set regards on the scores of a basic statistics subject, consisting in 216 students who attended this course in the first and second semeters of 2017 and 2018 at a Sourthern Brazilian university. In this particular application we considered only the final scores of approved students $(n=109)$.

As these scores range between $[6,10]$, the usual way to fit this problem is to use truncated models. Let $X$ a random variable that follows a continuous distribution with pdf $f(x, \theta)$ and $\operatorname{cdf} F(x, \theta)$. An easy way to truncate continuous models is taking

$$
\operatorname{Tr}(x, \theta)=\frac{g(x,)}{F(b)-F(a)}, \quad \text { for } \quad a<X \leq b,
$$

where $g(x, \theta)=f(x, \theta)$ when $a<X \leq b$ and zero, otherwise. All already known distributions can be easily truncated in the R software using the gamlss.tr package (STASINOPOULOS; RIGBY, 2016). Further, they can be fitted using the gamlss package.

The truncated distributions chosen here for comparisons are exponential (Exp), Weibull (Wei) and normal (NO). All distributions were truncated in the $a=6$ and $b=10$ break points and their pdf are given by (Gaussian pdf is omitted)

$$
\operatorname{Exp}: f(x)=\frac{1}{\mu} \exp \left[-\frac{x}{\mu}\right]
$$

and

$$
\text { Wei : } f(x)=\frac{\sigma x^{\sigma-1}}{\mu^{\sigma}} \exp \left[-\left(\frac{x}{\mu}\right)^{\sigma}\right] \text {. }
$$

The estimation of the parameters of all fitted distributions are summarized in Table 2. Additionally, the values of the Akaike Information Criterion (AIC) and Bayesian Information Criterion (BIC) statistics are also presented to compare the goodness-of-fit. All computational procedures for EU model were carried out using the optim function in R. For the competitive models, the MLEs were obtained using the packages mentioned before. The results indicate that the EU model has the lowest AIC and BIC (101.5 and 104.2, respectively) when confronted with all the fitted distributions, and therefore it could be chosen as the best model.

Table 2 - MLEs of models parameters, AIC and BIC measures for the scores data.

\begin{tabular}{llcc}
\hline Model & Parameters & AIC & BIC \\
\hline EU & $\hat{\alpha}=0.328$ & 101.5 & 104.2 \\
Wei & $\hat{\mu}=0.130, \hat{\sigma}=0.487$ & 240.2 & 245.6 \\
Exp & $\hat{\mu}=1.363$ & 239.5 & 242.2 \\
NO & $\hat{\mu}=-5.771, \hat{\sigma}=4.296$ & 242.9 & 248.3 \\
\hline
\end{tabular}

Source: The Authors.

Figure 3 displays the estimated densities of the fitted models presented on Table 2. Clearly, the EU distribution provides a closer fit to the histogram than the other models. Using some properties of the EU model presented above, we can conclude that: the average and variance of the score of the approved students are $E(X)=6.98$ and $\operatorname{Var}(X)=$ 1.27 , respectively; the confidence interval of $\alpha$ parameter is $0.328 \pm 0.062$.

Figure 3-Comparison of the fitted densities to the scores data.

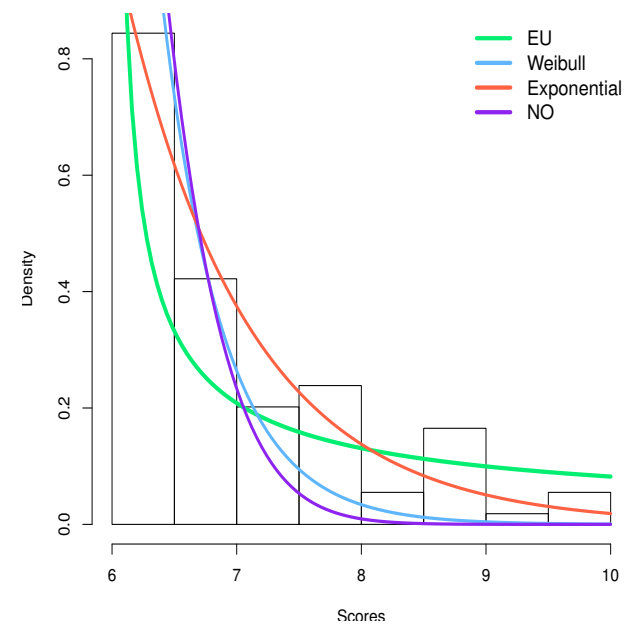

Source: The Authors. 


\section{Computational details}

To help R users use the proposed model, all codes used in this paper can be downloaded at $\mathrm{R}$ using the code

>source("https://git.io/fjinW")

After this step, the functions $\mathrm{dEU}(\mathrm{x}, \alpha, a, b)$ (density), $\mathrm{pEU}(\mathrm{x}, \alpha, a, b)$ (cdf), $\mathrm{qEU}(\mathrm{x}, \alpha, a, b)$ and $\operatorname{rEU}(\mathrm{n}, \alpha, a, b)$ (generate random samples) will be available. Note that the notation of these functions are the same of the others already implemented in R. We also provide the $\operatorname{mleEU}(\mathrm{y}, \alpha=1, a=0, b=1)$ function that can be used to easily obtain the MLEs, standard error, confidence boundaries, and the AIC and BIC statistics. To exemplify, see below an example about how to find the MLEs using a simulated random sample. This process was used to conduct the simulation study.

$>\mathrm{y}=\operatorname{rEU}(100, \mathrm{al} \mathrm{pha}=1, \mathrm{a}=0, \mathrm{~b}=10)$

$>\operatorname{mleEU}(\mathrm{y}, \mathrm{a}=0, \mathrm{~b}=10)$

\section{Conclusion}

We introduced and studied the exponentiated uniform (EU) distribution, which is indicated to fit variables with limited support. Various properties of the distribution were derived and investigated and a simulation study was conducted. A real data set demonstrated that the EU model can produce better fits than other usual models.

\section{Appendices}

\section{Appendix A.1}

Proof. The mean deviation about the mean (" $\delta_{1}$ ") is determined from (9). Replacing $\mu_{1}^{\prime}$ by (8) and the cdf by (4), $\delta_{1}$ can be expressed by

$$
\delta_{1}=2\left(\frac{\alpha b+a}{\alpha+1}\right)\left[\left(\frac{\mu-a}{b-a}\right)^{\alpha}-1\right]+2 T(\mu) .
$$

Replacing $\mu$ again and after some algebra, we have

$$
\delta_{1}=2 \frac{(\alpha b+a)}{(\alpha+1)^{\alpha+1}}\left[\alpha^{\alpha}-(\alpha+1)^{\alpha}\right]+2 T(\mu) .
$$

We can obtain $T(\mu)$ as

$$
T(\mu)=\int_{\mu}^{b} x \frac{\alpha}{(b-a)^{\alpha}}(x-a)^{\alpha-1} d x
$$

$$
\begin{aligned}
& =b-\frac{\mu(\mu-a)^{\alpha}}{(b-a)^{\alpha}}+\frac{(\mu-a)^{\alpha+1}}{(b-a)^{\alpha}(\alpha+1)} \\
& -\frac{b-a}{\alpha+1} .
\end{aligned}
$$

Replacing $\mu_{1}^{\prime}$ by (8) in the last equation, we have

$$
T(\mu)=b-\frac{(\alpha b+a) \alpha^{\alpha}}{(\alpha+1)^{\alpha+1}}+\frac{\alpha^{\alpha+1}(b-a)}{(\alpha+1)^{\alpha+1}}-\frac{b-a}{\alpha+1} .
$$

Inserting the last eq. in equation (15) and after some algebras the mean deviations about the mean can be expressed as

$$
\delta_{1}=\frac{2 \alpha^{\alpha+1}(b-a)}{(\alpha+1)^{\alpha+1}} .
$$

Proof. The mean deviations about median " $M$ " determined from (10). Replacing $\mu_{1}^{\prime}$ by (8), $\delta_{2}$ can be expressed by

$$
\delta_{2}=2 T(M)-\left(\frac{\alpha b+a}{\alpha+1}\right)
$$

Calculating the integrate $T(M)$ we have

$$
\begin{aligned}
T(M)= & \int_{M}^{b} x \frac{\alpha}{(b-a)^{\alpha}}(x-a)^{\alpha-1} d x \\
= & b-\frac{M(M-a)^{\alpha}}{(b-a)^{\alpha}}+\frac{(M-a)^{\alpha+1}}{(b-a)^{\alpha}(\alpha+1)} \\
& -\frac{b-a}{\alpha+1} .
\end{aligned}
$$

Replacing $M$ by the qf (5) and setting $u=0.5$ in (17) and after some algebra, we have

$$
\begin{aligned}
T(M)= & b\left(1-0.5^{\frac{1}{\alpha}+1}\right)+a\left(0.5+0.5^{\frac{1}{\alpha}+1}\right) \\
& +\frac{(b-a)\left(0.5^{1+\frac{1}{\alpha}}-1\right)}{\alpha+1} .
\end{aligned}
$$

Inserting (18) in (16) and after some algebra, the mean deviations about the mean can be expressed as

$$
\delta_{2}=\frac{2 a+\alpha a\left(1+0.5^{\frac{1}{\alpha}}\right)+\alpha b\left(1-0.5^{\frac{1}{\alpha}}\right)}{\alpha+1} .
$$

\section{Appendix A.2}

Proof. The Bonferroni curve is determined from (11). Replacing $E(X)$ by (8), the Bonferroni curve can be expressed by

$$
B(p)=\frac{(\alpha+1)}{p(\alpha b+a)} \int_{a}^{q} x \frac{\alpha}{(b-a)^{\alpha}}(x-a)^{\alpha-1} d x .
$$


By integration, we have

$$
\begin{aligned}
& \int_{a}^{q} x \frac{\alpha}{(b-a)^{\alpha}}(x-a)^{\alpha-1} d x= \\
& \frac{q(q-a)^{\alpha}}{(b-a)^{\alpha}}-\frac{(q-a)^{\alpha+1}}{(b-a)^{\alpha}(\alpha+1)} .
\end{aligned}
$$

Replacing the qf (5) in (19), we have

$$
\int_{a}^{q} x \frac{\alpha}{(b-a)^{\alpha}}(x-a)^{\alpha-1} d x=\frac{p\left(\alpha p^{\frac{1}{\alpha}}+a \alpha+a\right)}{\alpha+1} .
$$

Applying (20) in (19) and after some algebra, the Bonferroni curve can be expressed as

$$
B(p)=\frac{\alpha p^{\frac{1}{\alpha}}+a \alpha+a}{\alpha b+a} .
$$

Proof. Similar to the Bonferroni curve, the Lorenz curve defined in equation (11) can be expressed, replacing $E(X)$ by (8), by

$$
L(p)=\frac{(\alpha+1)}{(\alpha b+a)} \int_{a}^{q} x \frac{\alpha}{(b-a)^{\alpha}}(x-a)^{\alpha-1} d x .
$$

Using the integral in (20) and applying in (21), the Lorenz curve can be written as

$$
L(p)=\frac{p\left(\alpha p^{\frac{1}{\alpha}}+a \alpha+a\right)}{\alpha b+a} .
$$

\section{References}

ALJARRAH, M. A; LEE, C.; FAMOYE, F. On generating T-X family of distributions using quantile functions. Journal of Statistical Distributions and Applications, Heidelberg, v. 1, p. 1-17, 2014.

BOURGUIGNON, M.; SILVA, R. B.; CORDEIRO, G. M. The Weibull-G Family of Probability Distributions. Journal of Data Science, New York, v. 12, p. 53-68, 2014.

CORDEIRO, G. M.; ORTEGA, E. M. M.; CUNHA, D. C. C. The Exponentiated Generalized Class of Distributions. Journal of Data Science, New York, v. 11, p. 1-27, 2013.

GUPTA, R. C.; GUPTA, P. L.; GUPTA, R. D. Modeling failure time data by Lehman alternatives. Communications in Statistics, Theory and Methods, New York, v. 27, p. 887904, 1998.
GUPTA, R. D.; KUNDU, D. Exponentiated exponential family: an alternative to Gamma and Weibull distributions. Biometrical Journal, Weinheim, v. 43, p. 117-130, 2001.

LEE, C.; WON, H.Y. Inference on reliability in an exponentiated uniform distribution. Journal of the Korean Data \& Information Science Society, Gyeongsan-si, v. 17, p. 507-513, 2006.

NADARAJAH, S.; KOTZ, S. The exponentiated type distributions. Acta Applicandae Mathematicae, Heidelberg, v. 92, p. 97-111, 2006b.

PESCIM, R. R.; CORDEIRO, G. M.; DEMÉTRIO, C. G. B.; ORTEGA, E. M. M.; NADARAJAH, S. The new class of Kummer beta generalized distributions. SORT, Barcelona, v. 36, p. 153-180 2012.

RÉNYI, A. On the measures of entropy and information. Proceedings of Fourth Berkeley Symposium on Mathematics, Statistics and Probability, Berkeley, v. 1, p. 547-561, 1961.

RISTIC, M. M.; BALAKRISHNAN, N. The gamma exponentiated exponential distribution. Journal of Statistical Computation and Simulation, Philadelphia, v. 82, p. 11911206, 2012.

SHANNON, C. E. Prediction and Entropy of Printed English. Bell System Technical Journal, New York, v. 30, p. 50-64, 1951.

STASINOPOULOS, M.; RIGBY, B. gamlss. tr: Generating and Fitting Truncated gamlss.family Distributions. 2016. Available: <https://CRAN.R-project.org/package= gamlss.tr.> Access in: 01/05/2019.

TORABI, H.; MONTAZERI, N.H. The Logistic-Uniform distribution and its applications. Communications in Statistics - Simulation and Computation, New York, v. 43, p. 2551-2569, 2014.

TORABI, H.; MONTAZERI, N.H. The Gamma-Uniform distribution and its applications. Kybernetika, Prague, v. 48, p. 16-30, 2012

ZOGRAFOS, K.; BALAKRISHNAN, N. On families of beta- and generalized gamma-generated distributions and associated inference. Statistical Methodology, Amsterdam, v. 6, p. 344-362, 2009.

Received: May 17, 2019 Accepted: Aug. 26, 2019 